\title{
Mathematical Model of Financial Investment Risk
}

\section{Deyu Yin}

Rizhao Polytechnic, Rizhao, China

Email: yindeyu@163.com

How to cite this paper: Yin, D.Y. (2018) Mathematical Model of Financial Investment Risk. Journal of Mathematical Finance, 8, 127-136.

https://doi.org/10.4236/jmf.2018.81011

Received: January 17, 2018

Accepted: February 11, 2018

Published: February 14, 2018

Copyright $\odot 2018$ by author and Scientific Research Publishing Inc. This work is licensed under the Creative Commons Attribution International License (CC BY 4.0).

http://creativecommons.org/licenses/by/4.0/

\section{cc) (i) Open Access}

\begin{abstract}
This paper establishes the income and risk model in financial investment based on multi-objective programming theory, aiming to analyze the relationship between risk and return in financial investment and discuss the relationship between the risk the investor shall bear and decentralization degree of investment project. MATLAB software is used to analyze the investor's optimized return under fixed risk level and the minimized risk with defined benefit. In addition, it chooses the optimal portfolio under such risk level with respect to the bearing capacity of different risks. This paper performs sensitivity analysis of risk in income model using LINGO software, and puts forward the optimal portfolio for the investor without special preference. Calculations show that the model established is satisfactory in determining the optimal portfolio.
\end{abstract}

\section{Keywords}

Multi-Objective Programming, Risk Measurement, Income Level, Sensitivity Analysis, Optimal Portfolio

\section{Introduction}

Every investor cares about the risk and return of his/her investment in market economy. Compared with the projects in general, venture investment is characterized by the high investment, high risk, high return, in stages, timeliness and other features. Many traditional items and new risk factors which haven't appeared in investment will be produced in the investment process due to these characteristics. The new factors make the investors not only perform the conventional investment feasibility analysis when choosing project, but also discuss the source and constitution of the project implementation risk in-depth, and estimate the probability of risk and the potential damage to investor, so as to 
compare different programs and determine whether it is worth investing. As a result, the scientific, systematic and practical assessment system of risk investment is needed, and the study on risk item assessment system seems to be urgent and necessary [1].

\section{Briefing of the Question}

There is certain risk in financial investment. Generally speaking, the higher the expected return of risk investment, the greater the risk. Investors often pursue the higher expected return rate, avoid too much risk, and gain high return. Investors often make investment with different assets. There are $\mathrm{n}$ kinds of assets $S(i=1, \cdots, n)$ in the market for investors to choose. A considerable amount of money $\mathrm{M}$ of a company can be used to invest in a period of time. The company evaluated these $n$ kinds of assets and worked out that the average rate of return of $S_{i}$ within this period is $r_{i}(\%)$ and the rate of risk loss for purchasing $S_{i}$ is $q_{i}(\%)$. The more dispersion the investment, the lower the overall risk will be. As a result, the overall risk can be measured by the greatest risk of the invested $S_{i}$ if several kinds of assets are purchased by the money.

To purchase $S_{i}$, the transaction fee should be paid, with the rate being $p_{i}(\%)$, and when the purchase volume does not exceed the given value of $u_{i}$, the transaction fee should be calculated as per the volume that purchases $u_{i}$. In addition, suppose the bank deposit rate in the corresponding period is $r_{0}(\%)$, and there is no risk and no transaction fee.

Try to design an investment plan for this company, namely to choose to purchase several kinds of assets or deposit in a bank using the given money to maximize the net return and minimize the overall risk [2] [3].

Obtain a satisfactory program via calculation and analysis using the given data, and extend to the general situation. The following are the three cases which should be considered in practice.

1) Fix the risk level and optimize the returns

2) Fix the profitability and minimize the risk

3) Integrate the objective function and constraint condition

Different investors have different emphasizes on returns and risk, but it is normal within certain range. As a result, we can only request a program as good as possible, namely a program with minimized risk and maximized return, and that is in line with the mentality of general investors.

The respective average rate of return, the risk loss rate and transaction fee rate of the four given investment projects are different from each other. The calculation amount of transaction fee is a piecewise function, which is not easy to deal with in actual computation. But we notice that the value of $u_{i}$ is very small, $\sum u_{i}=103+198+52+40=387$ yuan, as for the largest $u_{i}, u_{2}=198<200$ yuan, $M$ is a quite large sum of money, and the value of transaction fee rate is also very small, even though $x_{i}<u_{i}$, the transaction fees calculated by $u_{i}$ and $x_{i}$ are almost the same. Therefore, in order for simplification, the constraint of 
$u_{i}$ will not be considered temporarily for the specific calculation below, and the transaction fee will be calculated using $x_{i}$, with the small error being analyzed in the discussion below.

With respect to the five kinds of investment, the bank is risk-free, the return $r_{0}=5 \%$ is a definite value, which will remain unchanged during the period of investment, but the return may be greater than the bank rate. Establish a model which is applicable to general investors, and put forward multiple investment programs that are suitable to various kinds of people according to their risk tolerance.

We have known from the previous analysis that the higher the risk, the greater the benefits. Therefore, the risk and benefit is a pair of contradictions. Establish the model using multi-objective, strive to pursue great profits and low risks, and seek a satisfactory solution.

Assume the proportion of the $I^{\text {th }}$ asset investment amount in the total amount is $x_{i}$, then the net return when the investment period expires is $\sum_{i}\left(x_{i} r_{i}-x_{i} p_{i}\right)$, and this is a two-goal linear programming model.

\section{Concept of Objective Programming}

1) Multi-objective programming is one of the mathematical programming methods in operational research. Establish relevant concepts of objective programming mathematical model. [4]

Assume $x_{1}, x_{2}$ are decision variables, and introduce in the positive and negative deviation variables of $d^{+}, d^{-}$

Positive deviation variable of $d^{+}$represents the part of decision value exceeding the target value; the negative deviation variable of $d^{-}$represents the part of decision value lagging behind the target value. Therefore, decision value cannot reach the condition simultaneously, namely it reaches the target value and lags behind the target value simultaneously, and there is constant $d^{+} \times d^{-}=0$.

2) Absolute constraint and object constraint

Absolute constraint refers to the equality constraint and inequality constraint that should be satisfied strictly; for example all the constraint conditions of linear programming, the solution that cannot meet these conditions is non-feasible solution, so they are hard constraints. Object constraint is specific to objective programming, and the right item of constraint can be regarded as the target value to be pursued. Positive or negative deviation is allowed when this target value is reached, so the positive and negative deviation variables are added in these constraints, and they are soft constrains [5].

3) Priority factor (priority) and weight coefficient

One programming problem often has several goals. However, the decision maker has primary or secondary priority when requesting the achievement of these objectives, requesting giving priority factor $P_{1}$ to the first one that reaches the target, giving priority factor $P_{2}, \cdots$, to the second one that reaches the 
target, and stipulates $P_{k} \gg P_{k+1}, k=1,2, \cdots, K$, indicating that $P_{k}$ has greater priority than $P_{k+1}$, namely ensure the realization of $P_{1}$ level target, and the second target may not be considered at such time; the $P_{2}$ level target is considered on the basis of $P_{1}$ level target; and so on in a similar fashion.

4) Objective function of objective programming

The objective function of objective programming (criterion function) is formed according to the positive and negative deviation variables of various object constraints and corresponding priority factors that have been given. When every target value is determined, the decision maker can require reducing the deviation from target value as much as possible. As a result, the objective function of objective programming can only be $\min z=f\left(d^{+}, d^{-}\right)$. It has three kinds of basic forms:

1) It is required to reach the target value exactly, namely make the positive and negative deviation variable as small as possible, at this time

$$
\min z=f\left(d^{+}+d^{-}\right)
$$

2) It is required not to exceed the target value, namely the target value is allowed not to be reached, and the positive deviation variable shall be as small as possible.

$$
\min z=f\left(d^{+}\right)
$$

3) It is required to exceed the target value, namely the exceeded amount is unlimited, but the negative deviation variable shall be as small as possible

$$
\min z=f\left(d^{-}\right)
$$

With respect to each specific target question, the objective function and the like can be constructed according to the decision maker's requirement and the priority factor granted to each target.

It is the programming method developed on the basis of the mathematical programming method of operational research--linear programming method, in order to overcome the defects of linear programming method, such as single objective function, rigor constraint condition, and the programming problem cannot be solved under contradiction conditions. The programming problem with multi-objective can be solved using such model. It takes seeking the minimum deviation variable between actual and object or resource limit as the starting point for constructing objective function, solves the problems relating to the balance of various targets in enterprises' production and operation, so it is widely used. Introduce the objective programming method into the decision of capital structure, try hard to operate this decision under the condition of considering multiple impact factors of the enterprise, and make the decision result more realistic.

In many practical problems, it is often hard to measure whether a program is good or not using just one index, in other words, it requires more than one target, and these targets are not often coordinate, event contradict [6] [7] [8] [9].

In the objective programming, some pursue minimum, and some pursue 
maximum, while maximum and minimum can be converted to each other. Therefore, it is easy for us to unify the multi-objective optimizing model as the general form:

Decision Variable: $x_{1}, \cdots, x_{n}$

Objective Function:

$$
\min f_{1}\left(x_{1}, \cdots, x_{n}\right)
$$

$\min f_{p}\left(x_{1}, \cdots, x_{n}\right)$
Constraint Condition: $\left\{\begin{array}{c}g_{1}\left(x_{1}, \cdots, x_{n}\right) \geq 0 \\ \vdots \\ g_{m}\left(x_{1}, \cdots, x_{n}\right) \geq 0\end{array}\right.$

In the model, it is not to consider to minimize or maximize each target, but to hope that each target can close to the given target under the limit of constraint condition. Therefore, we can give some constraint conditions, to make multiobjective programming into a single-objective linear programming, so as to obtain the optimal solution and conduct discussion.

\section{Model Assumption}

1) $M$ is the total investment, suppose $M=1$

2) The more dispersion the investment, the lower the risk

3) The highest risk ratio measurement in overall risk investment project $S_{i}$

4) $S_{i}(i=1, \cdots, n)$ is relatively independent

5) In the phase of investment, various indexes are stable, the rate of return of each index $r_{i}$, risk loss rate $q_{i}$, and transaction fee rate $p_{i}$ do not fluctuate much in the period of investment

6) Suppose the investment in a single asset $S_{i}$ has no upper limit, then the total asset $M$ can be invested into it.

7) In order for easy unified processing, the bank deposit can be regarded as the investment of asset $S_{0}$, its average rate of return is $r_{0}$, transaction fee rate is 0 , and risk loss is 0 .

8) Net return and overall risk are influenced by $r_{i}, p_{i}, q_{i}$

Symbol stipulation $S_{i}$ : the $i^{\text {th }}$ asset, $i=1,2, \cdots, n ; x_{i}$ : the investment volume into asset $S_{i}$ (excluding transaction fee) $(i=0, \cdots, n) ; r_{i}$ : the average rate of return of the investment into asset $S_{i}(i=0, \cdots, n) ; q_{i}$ : the risk loss rate when making investment into asset $S_{i}(i=0, \cdots, n) ; p_{i}$ : the transaction fee rate when making investment into asset $S_{i}(i=0, \cdots, n) ; u_{i}$ : the transaction fee volume (fixed value) when making investment into asset into asset $S_{i}(i=0, \cdots, n) ; r_{0}$ : bank interest rate in the corresponding period; $R_{i}$ : net return of $S_{i} ; f$ : overall risk; $x_{i}$ : capital of investment project $S_{i}$; a: investment risk; $Q$ : overall return; $\Delta Q$ : increment of overall return

\section{Model Establishment}

We discuss the following cases respectively: 
1) The overall risk is measured by the highest risk in the invested $S_{i}$, namely $\max \left\{x_{i} q_{i}, i=1,2, \cdots, n\right\}$.

2) When the company makes investment in the asset in the market, two standards measuring the investment program are involved, namely there are two goals: 1 . High net return; 2. Low risk

One piecewise function for paying transaction fee when purchasing $S_{i}$, namely

Transaction fee $=\left\{\begin{array}{l}p_{i} x_{i}, x_{i}>u_{i} \\ p_{i} u_{i}, x_{i} \leq u_{i}\end{array}\right.$

The given fixed value $u_{i}$ (unit: yuan) in the question is very small relative to the total asset $M, p_{i} u_{i}$ is smaller which can be ignored, thus the net return for purchasing $S_{i}$ is $R_{i}=\left(r_{i}-p_{i}\right) x_{i}$.

3) This is a multi-objective programming model to maximize net revenue and minimize the overall risk:

$$
\begin{aligned}
& \text { Object Function }\left\{\begin{array}{l}
\max \sum_{i=0}^{n}\left(r_{i}-p_{i}\right) x_{i} \\
\min \max \left\{q_{i} x_{i}\right\}
\end{array}\right. \\
& \text { Constraint Condition }\left\{\begin{array}{l}
\sum_{i=0}^{n}\left(1+p_{i}\right) x_{i}=M \\
x_{i} \geq 0, i=1, \cdots, n
\end{array}\right.
\end{aligned}
$$

4) In actual investment, the investors bear the risk of different degrees or the preference degree of risk is different. If a limit a is given, the corresponding in vestment program can be found if the highest risk $\frac{q_{i} x_{i}}{M} \leq a$. In this way, the multi-objective programming is changed into a single-objective programming [10].

Therefore, we obtain the following mathematical programming model

\subsection{Model I Fixes Risk Level and Optimizes the Revenue}

If we give a risk limit a, then the highest risk degree is $\frac{q_{i} x_{i}}{M} \leq a$, the fixed risk level is obtained. So in order to optimize the revenue, as mentioned above, it has been proved that the constraint of $u_{i}$ will not be considered, as it has little influence on the investment program. We can obtain the model by considering various cases:

Objective function $Q=\max \sum_{i=0}^{n}\left(r_{i}-p_{i}\right) x_{i}$

Constraint condition: s.t. $\left\{\begin{array}{l}\frac{q_{i} x_{i}}{M} \leq a \\ \sum_{i=0}^{n}\left(1+p_{i}\right) x_{i}=M \quad \text { Where, } M=1 \\ x_{i} \geq 0, i=0,1, \cdots, n\end{array}\right.$

This model is a single object linear programming. It is easy to get the optimal 
solution with the given value a.

\subsection{Model II Fixes Revenue Level and Minimizes Risk}

If the investor wish the total revenue reaches at least level $K$ and above, then seek corresponding investment portfolio when the risk is the lowest. We can establish the following model

Objective function $f=\min \max \left\{q_{i} x_{i}\right\}$

Constraint condition s.t. $\left\{\begin{array}{l}\sum_{i=0}^{n}\left(r_{i}-p_{i}\right) x_{i} \geq K \\ \sum_{i=0}^{n}\left(1+p_{i}\right) x_{i}=M \quad \text { Where, } M=1 \\ x_{i} \geq 0, i=0,1, \cdots, n\end{array}\right.$

\section{Model Calculation}

We obtain the relevant data of four kinds of assets $S_{1}, \cdots, S_{4}$ and bank $S_{0}$ data as follows through data collection (Table 1):

\subsection{Numerical Calculation of Model I}

Model I is:

$$
\begin{aligned}
& \min f=(-0.05,-0.27,-0.19,-0.185,-0.185)\left(x_{0}, x_{1}, x_{2}, x_{3}, x_{4}\right)^{\mathrm{T}} \\
& \text { s.t. }\left\{\begin{array}{l}
x_{0}+1.01 x_{1}+1.02 x_{2}+1.045 x_{3}+1.065 x_{4}=1 \\
0.025 x_{1} \leq a \\
0.015 x_{2} \leq a \\
0.055 x_{3} \leq a \\
0.026 x_{4} \leq a \\
x_{i} \geq 0, i=0,1, \cdots, 4
\end{array}\right.
\end{aligned}
$$

Different investors have different risk degree as $a$ is a randomly given risk degree without a principle. We search cyclically starting from $a=0$ with $\Delta a=0.002$ as the step size, and conduct programming using matlab with the operation results as follows (Figure 1):

\section{Result analysis:}

1) Risk is high and the revenue is also high.

Table 1. Actual data of the investment in various assets.

\begin{tabular}{ccccc}
\hline$S_{i}$ & $r_{i}(\%)$ & $q_{i}(\%)$ & $p_{i}(\%)$ & $u_{i}($ yuan $)$ \\
\hline$S_{0}$ & 5 & 0 & 0 & 0 \\
$S_{1}$ & 28 & 2.5 & 1 & 103 \\
$S_{2}$ & 21 & 1.5 & 2 & 198 \\
$S_{3}$ & 23 & 5.5 & 4.5 & 52 \\
$S_{4}$ & 25 & 2.6 & 6.5 & 40 \\
\hline
\end{tabular}




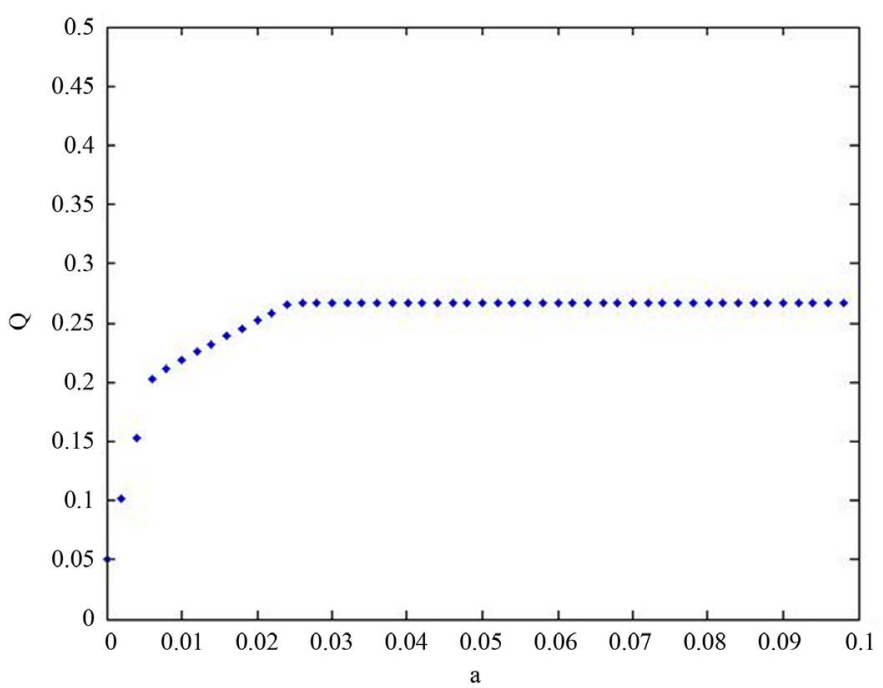

Figure 1. Relationship of this model I risk measurement and revenue.

2) The more dispersion the investment, the lower risk the investor will bear, which is in line with the meaning of the question. In other words, the adventurous investor will concentrate his/her investment, while the conservative investor will disperse the investment as much as possible.

3) Any point on the curve represents the maximum possible return under such risk level and the lowest risk required by such return. With respect to the bearing capacity of different risks, choose the optimal portfolio under such risk level [11].

4) There is a turning point near $a=0.006$. At the left side of this point, the return increases very fast when risk increases little. At the right side of this point, the return increases very slow when the risk increases rapidly [9]. As a result, with respect to the investor having no special preference of risk and return, the inflection point of the curve shall be selected as the optimal portfolio, which is about $a^{*}=0.6 \%$ and $Q^{*}=20 \%$ and the corresponding investment program as follow (Table 2):

\subsection{Numerical Implementation of Model II}

Feed in the investigated data, Model II becomes the linear programming:

$$
\begin{aligned}
& \min f=(1,0,0,0,0,0)\left(t, x_{0}, x_{1}, x_{2}, x_{3}, x_{4}\right)^{T} \\
& \text { s.t. }\left\{\begin{array}{l}
x_{0}+1.01 x_{1}+1.02 x_{2}+1.045 x_{3}+1.065 x_{4}=1 \\
0.05 x_{0}+(0.28-0.01) x_{1}+(0.21-0.02) x_{2} \\
\quad+(0.23-0.045) x_{3}+(0.25-0.065) x_{4} \geq K \\
0.025 x_{1} \leq t \\
0.015 x_{2} \leq t \\
0.055 x_{3} \leq t \\
0.026 x_{4} \leq t \\
t, x_{i} \geq 0, i=0,1, \cdots, 4
\end{array}\right.
\end{aligned}
$$


Different investors have different risk degree as $a$ is a randomly given risk degree without a principle. We search cyclically starting from $a=0$ with $\Delta a=0.002$ as the step size, and conduct programming using matlab with the operation results as follows (Figure 2):

\section{Result Analysis:}

1) Risk will increase when pursuing high return.

2) Any point on the curve represents the investment program with lowest risk when the revenue level is fixed. As for different minimum return requirement, select the corresponding optimal portfolio and the corresponding risk is the lowest at this time.

3) There is turning point near the revenue level of $K=20 \%$. At the left side of this point, the risk degree increases slowly when the revenue level requirement increases. But at the right side of this point, the risk degree increases obviously when the revenue level requirement increases [12] [13]. Therefore, with respect to the investors without special preference of risk and return, they should make rational investment and be on guard against the accompanying high risk, and should not pursue high return blindly. As for these several kinds of assets, the investment at revenue level of $K=20 \%$, the lowest risk is $0.59 \%$, and the corresponding strategy as follow (Table 3 ):

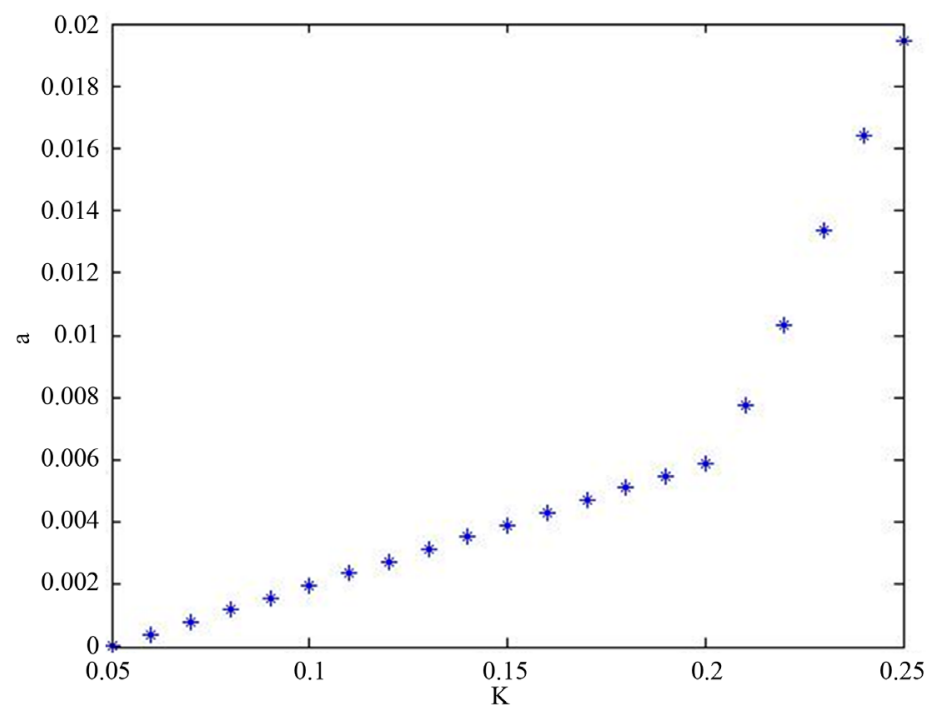

Figure 2. Diagram of relationship between revenue level and risk degree in Model II.

Table 2. Optimal portfolio of Model I.

\begin{tabular}{ccccccc}
\hline Risk Degree & Return & $\mathrm{x} 0$ & $\mathrm{x} 1$ & $\mathrm{x} 2$ & $\mathrm{x} 3$ & $\mathrm{x} 4$ \\
\hline 0.0060 & 0.2019 & 0 & 0.2400 & 0.4000 & 0.1091 & 0.2212 \\
\hline
\end{tabular}

Table 3. Optimal solution of Model II.

\begin{tabular}{ccccccc}
\hline Risk Degree & Return & $\mathrm{x} 0$ & $\mathrm{x} 1$ & $\mathrm{x} 2$ & $\mathrm{x} 3$ & $\mathrm{x} 4$ \\
\hline 0.0059 & 0.20 & 0 & 0.2350 & 0.3917 & 0.1068 & 0.2260 \\
\hline
\end{tabular}




\section{Conclusion}

This paper introduces relevant concept of multi-objective programming, and then establishes the return and risk model in financial investment, aiming to analyze the relationship between risk and return in financial investment, and discuss the relationship between the risk the investor shall bear and the dispersion degree of investment project. Then, the paper analyzes the optimized return of the investor under fixed risk level using MATLAB software. In addition, it also analyzes the minimized risk under fixed return, indicating that the optimal portfolio under this risk level shall be selected for the bearing capacity of different risks. It puts forward the optimal portfolio of investor without special preference of risk and return.

\section{References}

[1] Chen, Y.H., Xu, L. and Wang, X.G. (2000) Application of DEA in Risk Investment Project Decision. Science-Technology and Management.

[2] Peng, S.K. (2000) Capital Asset Pricing Model and Risk Project Evaluation. Forecasting.

[3] Yan, T.H. and Zhang, L. (2002) Analysis and Comparison of Risk Investment Project Pricing Methods. China Economist, No. 11.

[4] Vance, H. and Robert, D. (1994) Toward a Model of Venture Capital Investment Decision Making. Financial Management, 23, 8-37

[5] Trester, J.J. (1998) Venture Capital Contracting under Asymmetric Information Journal of Banking \& Finance, 22, 675-699.

[6] Li, D. (1982) Operational Research. Tsinghua University Press, Beijing.

[7] Zhou, H.L. (1995 Linear Programming and Its Application. Metallurgical Industry Press.

[8] Wang, J.F. (2000) Venture Investment Practice and Cases. Tsinghua University Press, Beijing.

[9] Wu, S.S. (1998) Application of Mathematical Method in Analysis of Financial Investment Risk. Journal of Huaqiao University (Humanities \& Social Science), No. 1.

[10] Krishnamurthy, P.K., Fisher, J.B. and Johnson, C. (2010) Mainstreaming Local Perceptions of Hurricane Risk into Policymaking: A Case Study of Community GIS in Mexico. Global Environmental Change, No. 1.

[11] Fan, Y.L., Ginis, I., Hara, T., Wright, C.W. and Walsh, E.J. (2009) Numerical Simulations and Observations of Surface Wave Fields under an Extreme Tropical Cyclone. Journal of Physical Oceanography, No. 9.

[12] Vickery, P.J., Wadhera, D., Powell, M.D. and Chen, Y.Z. (2009) A Hurricane Boundary Layer and Wind Field Model for Use in Engineering Applications. Journal of Applied Meteorology and Climatology, No. 2.

[13] Liu, X.Z. and Li, J.Z. (2008) Application of SCS Model in Estimation of Runoff from Small Watershed in Loess Plateau of China. Chinese Geographical Science, No. 3. 